\title{
Investigation of the influence of nuclear matter on hard neutrino nucleus interaction using the HARDPING Monte Carlo Event Generator
}

\author{
Ya.A. Berdnikov ${ }^{1,2}$, A. Ya. Berdnikov ${ }^{1}$, V.T. Kim ${ }^{1,2}$, A.E. Ivanov ${ }^{1}$, D.P. Suetin ${ }^{1,2, \star}$, and K.D. Tiangov ${ }^{1}$ \\ ${ }^{1}$ Peter the Great Saint-Petersburg Polytechnic University, St. Petersburg, 195251, Russia \\ ${ }^{2}$ The Petersburg Nuclear Physics Institute, National Research Center "Kurchatov Institute", Gatchina, \\ Leningrad district, 188300, Russia
}

\begin{abstract}
Hadron production in neutrino-nucleus interactions is implemented in Monte Carlo event generator HARDPING (HARD Probe INteraction Generator). Such effects as formation length, energy loss and multiple rescattering for produced hadrons and their constituents are taken into account in HARDPING. Available data from WA/59 and SCAT collaborations on hadron production in neutrino-nucleus collisions is described by HARDPING with a reasonable agreement.
\end{abstract}

Hadronization of quarks and gluons is one of the most intriguing processes of nonperturbative QCD. Use of nuclear targets may allow to study important features of space-time picture of hadronization, like hadron formation length and energy loss, see, e.g, for a review [1- 7] and references therein.

The understanding of quark propagation in nuclear medium is crucial to interpret nucleus-nucleus, proton-nucleus and lepton-nucleus interactions. To simplify interpretation of observable effects first of all let's consider hadron production in lepton scattering off nuclei.

It is well known from theoretical and experimental studies of lepton-nucleus collisions at high energies that hadrons are not produced at the point of collision but only after certain "formation" length [1]. In the Lund string fragmentation model, the production of hadrons is described as a two-stage process. On the first perturbative stage pre-hadron is formed at the end of the string. On the next nonperturbative stage hadron is formed. Prior to formation of pre-hadron, the knocked-out quark propagates through nuclear matter with a very small cross section. It takes some time to form a pre-hadron at the perturbative stage (formation time, $t_{p}$ or formation length, $l_{p}$ ). When the prehadron is formed, it interacts with nuclear matter with pre-hadron cross section, which is different from hadronic cross section. Also it takes an extra time to form the final hadron from pre-hadron state. Therefore, formation length consists of two parts $l_{p}$ and $l_{h}$, which correspond to pre-hadron and hadron stages accordingly. There are two approaches to calculate such formation length. The first one is based on a simplified description of nonperturbative stage [1], while the second one [3] is based on Lund string model, which neglects the energy loss effects during perturbative stage. Present work takes into account both effects: energy loss in perturbative stage and Lund string model hadronization in nonperturbative stage. In the approach presented in this article effect of energy loss was built into HARDPING [8-10] using PYTHIA event generator implementation of parton shower for the perturbative stage and Lund string model for the nonperturbative one.

^e-mail: suetindaniil@gmail.com 


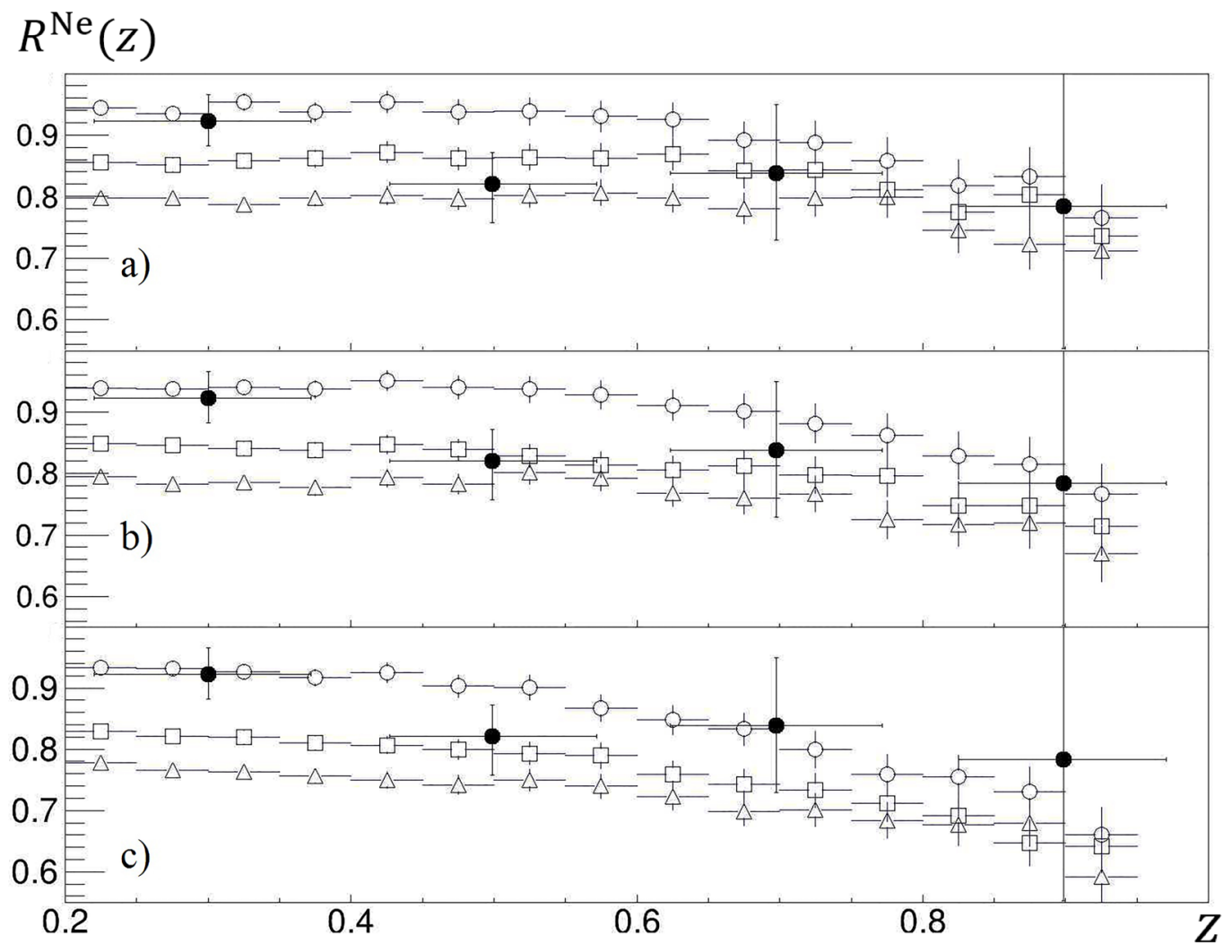

Figure 1. Ratio of hadron yield on neon (Ne) target to analogous yield on proton (p) target as function of $z$ for neutrino interactions (2) in kinematic region $4<v<8 \mathrm{GeV}$. Black circles represent WA/59 collaboration data [11], blank circles, squares and triangles represent HARDPING simulation data with string tension coefficient $\kappa$ equal to $1.5,2.5$ and $3.5 \mathrm{GeV} / \mathrm{fm}$ accordingly. Figure shows results of simulation with pre-hadron nucleon cross-section equal to a) $5 \mathrm{mb}$; b) $10 \mathrm{mb}$; c) $20 \mathrm{mb}$.

During the perturbative stage, which corresponds to formation length $l_{p}$, a pre-hadron state is formed. It can interact with intranuclear nucleons with inelastic pre-hadronic cross-section. At the end of the nonperturbative stage the observed hadron is formed. Produced pre-hadrons and hadrons can undergo soft collisions with intranuclear nucleons (with small momentum transfer: $|t| \sim 1 \mathrm{GeV}^{2}$ ). Therefore one has to take into account their soft multiple rescattering and energy loses in nuclear matter [8-10]. The goal of present work is to study these effects for neutrino-nuclei collisions using HARDPING.

The experimental data from WA/59 collaboration on hadron production in neutrino-nuclei collisions are presented in terms of nuclear modification factor $R_{\mathrm{Ne}}$, which represents ratio of hadron yield on neon (Ne) target to analogous yield on proton (p) target, as function of virtual boson energy $\left(v=E_{\mu}-E_{v}\right)$ or virtual boson energy fraction taken away by hadron $\left(z=E_{h} / v\right)$ :

$$
R^{\mathrm{Ne}}(v)=\frac{1}{N_{\mathrm{Ne}}} \frac{d N_{\mathrm{Ne}}^{c h}(v)}{d v} / \frac{1}{N_{\mathrm{p}}} \frac{d N_{\mathrm{p}}^{c h}(v)}{d v}
$$




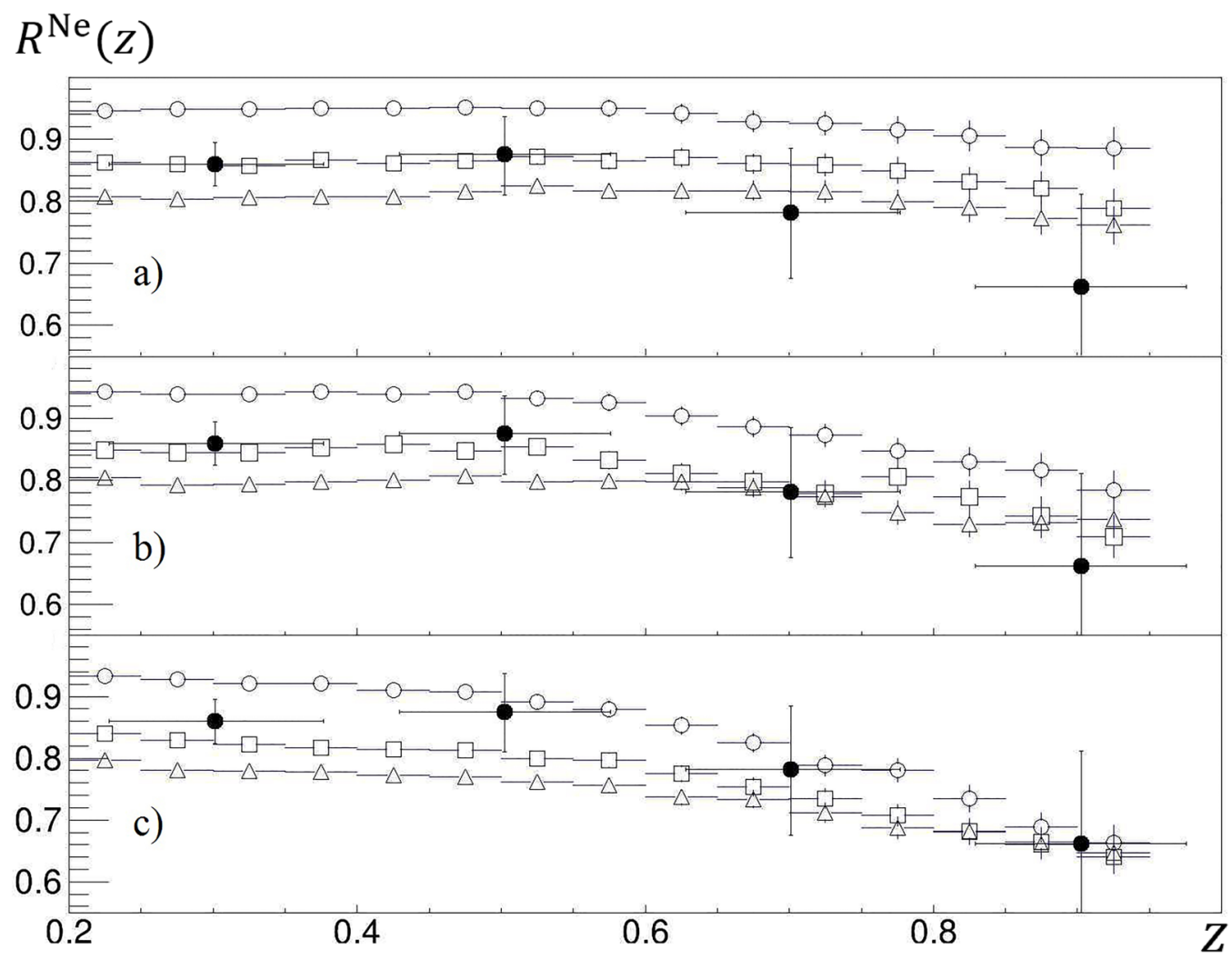

Figure 2. Ratio of hadron yield on neon $(\mathrm{Ne})$ target to analogous yield on proton (p) target as function of $z$ for anti-neutrino interactions (2) in the kinematic region $4<v<8 \mathrm{GeV}$. Black circles represent WA/59 collaboration data [11], blank circles, squares and triangles represent HARDPING simulation data with string tension coefficient $\kappa$ equal to $1.5,2.5$ and $3.5 \mathrm{GeV} / \mathrm{fm}$ accordingly. Figure shows results of simulation with pre-hadron nucleon cross-section equal to a) $5 \mathrm{mb}$; b) $10 \mathrm{mb}$; c) $20 \mathrm{mb}$.

$$
R^{\mathrm{Ne}}(z)=\frac{1}{N_{\mathrm{Ne}}} \frac{d N_{\mathrm{Ne}}^{c h}(z)}{d z} / \frac{1}{N_{\mathrm{p}}} \frac{d N_{\mathrm{p}}^{c h}(z)}{d z}
$$

where $N_{\mathrm{Ne}}$ and $N_{\mathrm{p}}$ are numbers of neutrino charged current interactions on Ne and p targets accordingly; $N_{\mathrm{Ne}}^{c h}$ and $N_{\mathrm{p}}^{c h}$ are yields of hadron on $\mathrm{Ne}$ and $\mathrm{p}$ targets accordingly.

Neutrino and anti-neutrino beams in WA/59 originate from $400 \mathrm{GeV}$ protons impinging on a beryllium target. Simulations of neutrino-nuclei collisions obtained by HARDPING were compared with WA/59 data [11]. The results are shown in figs. 1, 2, 3 .

Experimental results from SCAT collaboration on hadron production in neutrino-nuclei collisions are presented in terms of nuclear modification factor $R^{c h}$, which represents ratio of hadron yield on nuclear $(\mathrm{A}=28)$ target to analogous yield on deuterium (D) target, as function of virtual boson energy $\left(v=E_{\mu}-E_{v}\right)$ or virtual boson energy fraction taken away by hadron $\left(z=E_{h} / v\right)$ :

$$
R^{c h}(v)=\frac{1}{N_{\mathrm{A}}} \frac{d N_{\mathrm{A}}^{c h}(v)}{d v} / \frac{1}{N_{\mathrm{D}}} \frac{d N_{\mathrm{D}}^{c h}(v)}{d v}
$$




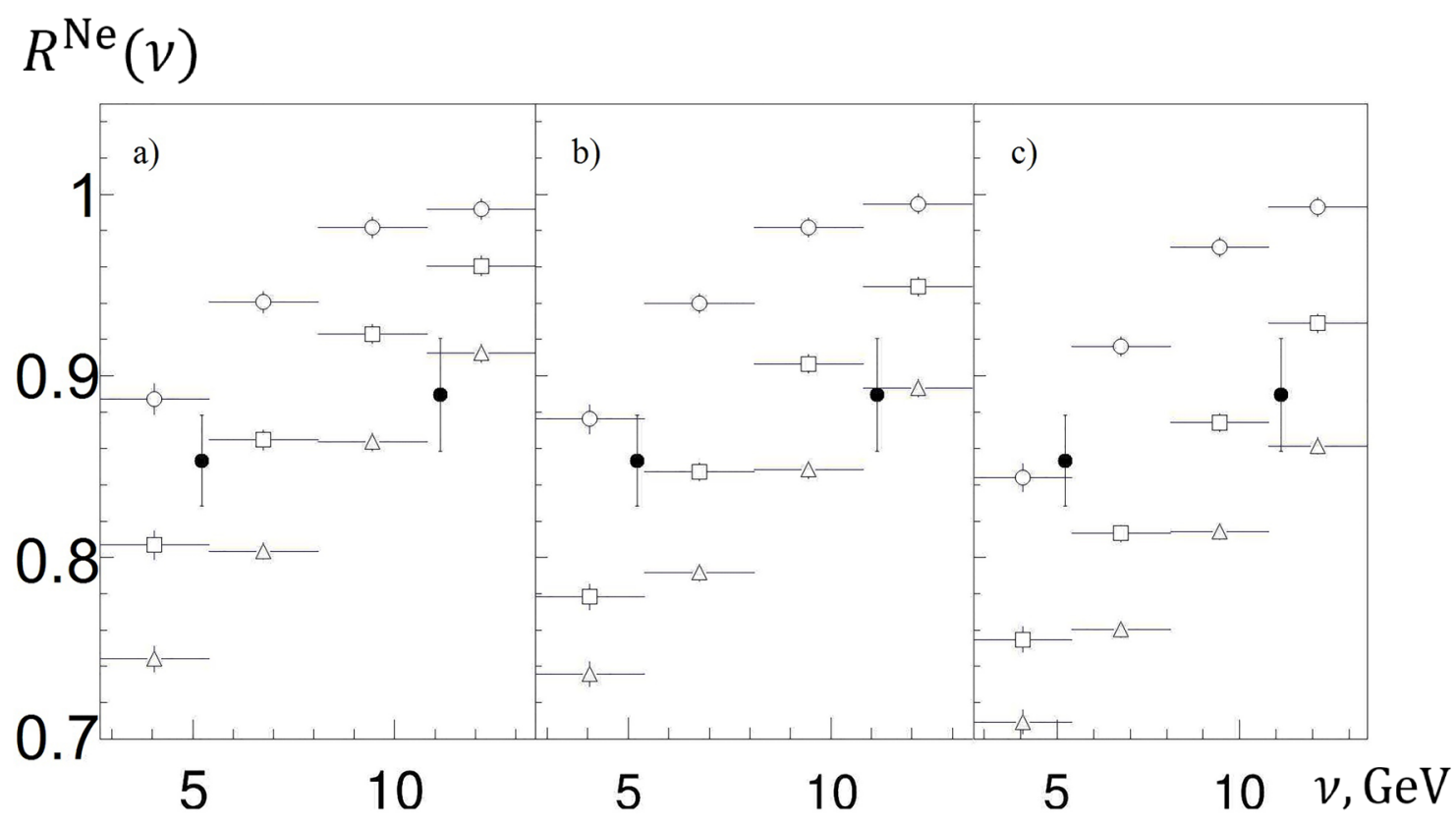

Figure 3. Ratio of hadron yield on neon (Ne) target to analogous yield on proton (p) target as function of $v$ for neutrino and anti-neutrino interactions (1) in the kinematic region $z>0.2$. Black circles represent WA/59 collaboration data [11], blank circles, squares and triangles represent HARDPING simulation data with string tension coefficient $\kappa$ equal to $1.5,2.5$ and $3.5 \mathrm{GeV} / \mathrm{fm}$ accordingly. Figure shows results of simulation with pre-hadron nucleon cross-section equal to a) $5 \mathrm{mb}$; b) $10 \mathrm{mb}$; c) $20 \mathrm{mb}$.

$$
R^{c h}(z)=\frac{1}{N_{\mathrm{A}}} \frac{d N_{\mathrm{A}}^{c h}(z)}{d z} / \frac{1}{N_{\mathrm{D}}} \frac{d N_{\mathrm{D}}^{c h}(z)}{d z}
$$

where $N_{\mathrm{A}}$ and $N_{\mathrm{D}}$ are numbers of neutrino charged current interactions on A and D targets accordingly, $N_{\mathrm{A}}^{c h}$ and $N_{\mathrm{D}}^{c h}$ are yields of hadron on $\mathrm{A}$ and $\mathrm{D}$ targets accordingly. Neutrino and anti-neutrino beams in SCAT originate from $70 \mathrm{GeV}$ protons. Simulations of neutrino-nuclei collisions obtained by HARDPING were compared with SCAT data [12]. The results are shown in Figs. 4 and 5.

Performed simulations show a reasonable agreement of MC HARDPING with experimental data from WA/59 and SCAT [11, 12]. This allows to fix values of such model parameters as pre-hadron nucleon cross-section $\sigma=10 \mathrm{mb}$ and string tension $\kappa=2.5 \mathrm{GeV} / \mathrm{fm}$, with which the best agreement with the two experiments was achieved. In conclusion HARDPING Monte Carlo event generator describes:

- Experimental data from WA/59 collaboration [11] on hadron production in neutrino- and antineutrino nuclei scattering at average incident particle energy of 54 and $40 \mathrm{GeV}$ accordingly;

- Experimental data from SCAT collaboration [12] on hadron production in neutrino-nuclei scattering at average neutrino energy of $10 \mathrm{GeV}$.

\section{References}

[1] B.Z Kopeliovich, J Nemchik, E. Predazzi, and A. Hayashigaki, Nucl. Phys. A 740, 211 (2004) 


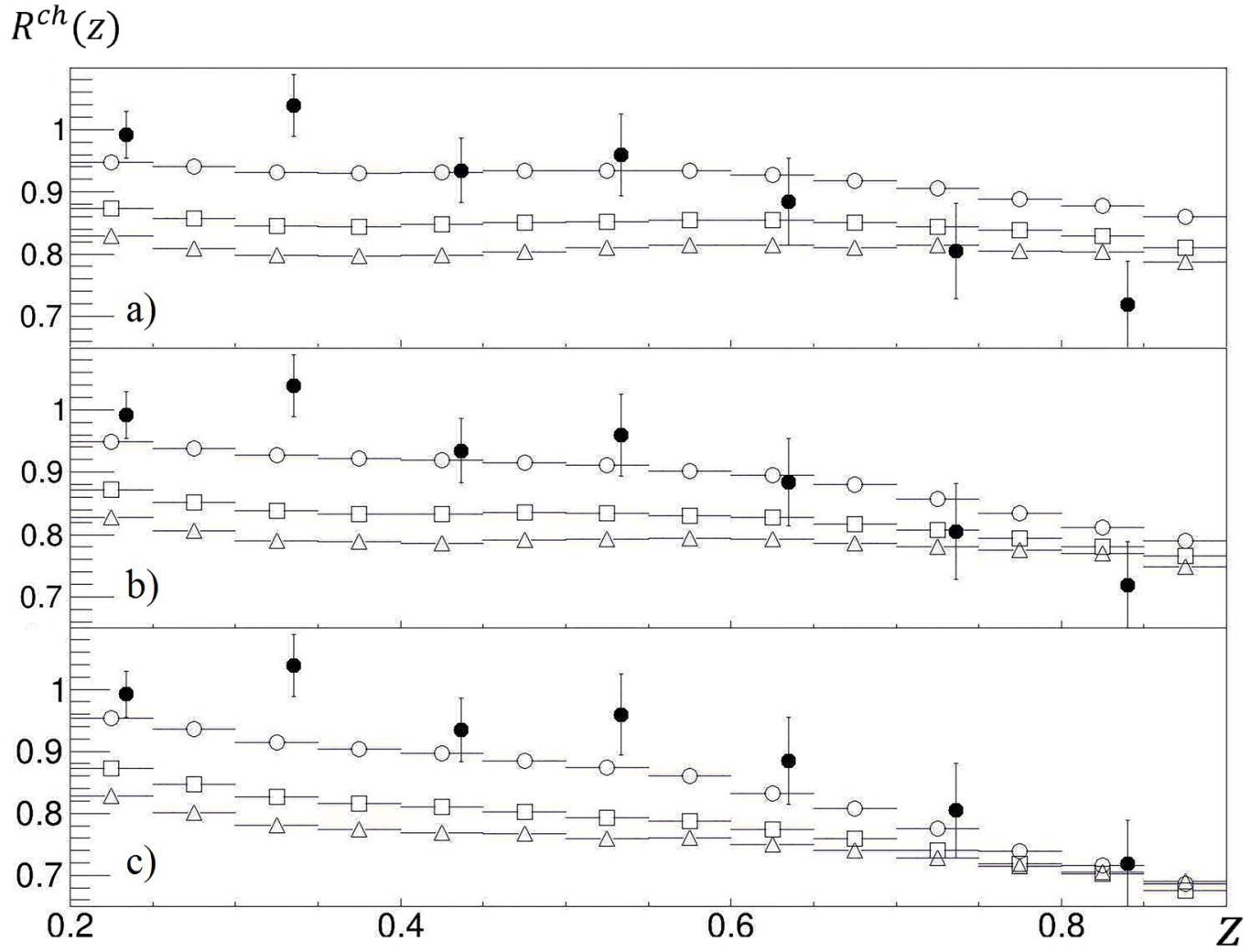

Figure 4. Ratio of hadron yield on nuclear $(\mathrm{A}=28)$ target to analogous yield on deuterium target as function of $z$ for neutrino interactions (4). Black circles represent SCAT collaboration data [12], blank circles, squares and triangles represent HARDPING simulation data with string tension coefficient $\kappa$ equal to $1.5,2.5$ and $3.5 \mathrm{GeV} / \mathrm{fm}$ accordingly. Figure shows results of simulation with pre-hadron nucleon cross-section equal to a) $5 \mathrm{mb}$; b) 10 $\mathrm{mb}$; ) $20 \mathrm{mb}$.

[2] S. Domdey, D. Grunewald, B.Z. Kopeliovich, Nucl. Phys. A 825, 200-211 (2009)

[3] A. Accardi, V. Muccifora, H.J. Pirner, Nucl. Phys. A 720, 131-157 (2003)

[4] A. Accardi, D. Grunewald, V. Muccifora, H.J. Pirner, Nucl. Phys. A 761, 67-96 (2005)

[5] R. Baier, D. Schiff and B. G. Zakharov, Ann. Rev. Nucl. Part. Sci. 50, 37 (2000)

[6] R. Baier, Y. L. Dokshitzer, A. H. Mueller et al., Nucl. Phys. B 484, 265 (1997)

[7] B. G. Zakharov, JETP Lett. 65, 615 (1997)

[8] Ya.A. Berdnikov, A.E. Ivanov, V.T. Kim, V.A. Murzin D.P Suetin, Nucl. Phys. B. Proc. Suppl. 245, 267-270 (2013)

[9] Ya.A. Berdnikov, A.E. Ivanov, V.T. Kim, D.P Suetin, Bulletin of the Russian Academy of Sciences: Physics 79(7), 925-929 (2015)

[10] Ya.A. Berdnikov, A.E. Ivanov, V.T. Kim, D.P Suetin, AIP Conference Proceedings 1701, 080005 (2016)

[11] W. Burkot, , T. Coghen, J. Czyżewski, Zeitschrift für Physik C70(1), 47-53 (1996) 


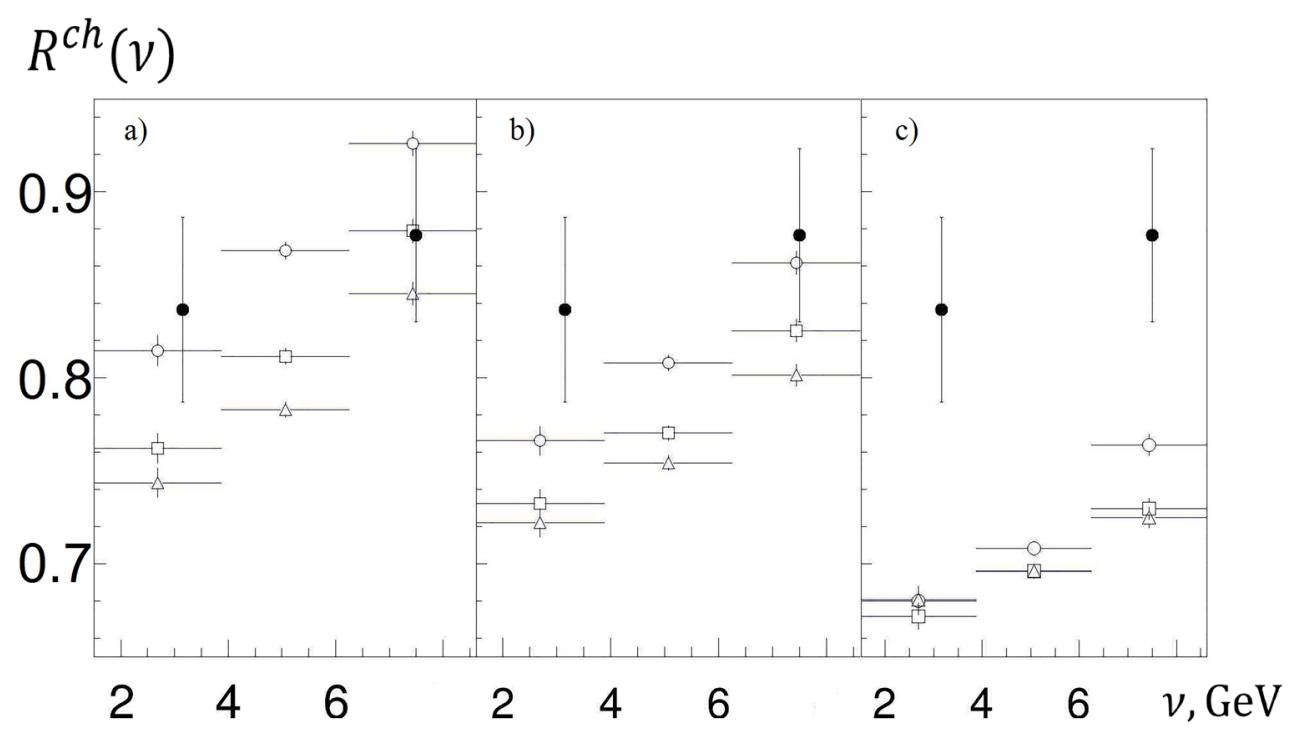

Figure 5. Ratio of hadron yield on nuclear $(\mathrm{A}=28)$ target to analogous yield on deuterium target as function of $v$ for neutrino and anti-neutrino interactions (3) in the kinematic region $z>0.2$. Black circles represent SCAT collaboration data [12], blank circles, squares and triangles represent HARDPING simulation data with string tension coefficient $\kappa$ equal to $1.5,2.5$ and $3.5 \mathrm{GeV} / \mathrm{fm}$ accordingly. Figure shows results of simulation with pre-hadron nucleon cross-section equal to a) $5 \mathrm{mb}$; b) $10 \mathrm{mb}$; c) $20 \mathrm{mb}$.

[12] N. M. Agababyan, V. V. Ammosov, M. R. Atayan, Physics of Atomic Nuclei 66, 310-318 (2003) 